\title{
Circulating Levels of Tissue Plasminogen Activator and Plasminogen Activator Inhibitor-1 Are Independent Predictors of Coronavirus Disease 2019 Severity: A Prospective, Observational Study
}

\author{
Brandon Michael Henry, MD ${ }^{1, *}$ Isaac Cheruiyot, BS ${ }^{2, *} \quad$ Justin L. Benoit, MD ${ }^{3}$ Giuseppe Lippi, MD ${ }^{4}$ \\ Zoltán Prohászka, MD, $\mathrm{PhD}^{5}$ Emmanuel J. Favaloro, PhD, FFSc (RCPA) ${ }^{6}$ Stefanie W. Benoit, MD ${ }^{7,8}$
}

${ }^{1}$ Cardiac Intensive Care Unit, The Heart Institute, Cincinnati Children's Hospital Medical Center, Cincinnati, Ohio

${ }^{2}$ School of Medicine, University of Nairobi, Nairobi, Kenya

${ }^{3}$ Department of Emergency Medicine, University of Cincinnati, Cincinnati, Ohio

${ }^{4}$ Section of Clinical Biochemistry, Department of Neuroscience, Biomedicine and Movement, University of Verona, Verona, Italy

${ }^{5}$ Department of Medicine and Hematology, Research Laboratory, Semmelweis University, Budapest, Hungary

${ }^{6}$ Haematology, Sydney Centres for Thrombosis and Haemostasis, Westmead Hospital, Institute of Clinical Pathology and Medical Research (ICPMR), NSW Health Pathology, Westmead, NSW, Australia

${ }^{7}$ Division of Nephrology and Hypertension, Cincinnati Children's Hospital Medical Center, Ohio

${ }^{8}$ Department of Pediatrics, University of Cincinnati College of Medicine, Ohio

Semin Thromb Hemost 2021;47:451-455

A substantial pool of evidence suggests that the pathophysiology of the severe form of coronavirus disease 2019 (COVID19 ) is mediated, in part, by a hypercoagulable state termed COVID-19-associated coagulopathy (CAC), ${ }^{1-3}$ and characterized by micro- and macrovascular thrombosis. ${ }^{4,5}$ Approximately $27 \%$ of critically ill intensive care unit (ICU)-admitted COVID-19 patients develop venous thromboembolism during admission, ${ }^{6}$ while $4.4 \%$ experience arterial thrombotic complications. ${ }^{7}$ Autopsy studies on COVID-19 patients have demonstrated extensive presence of microthrombi within pulmonary capillaries, associated with diffuse alveolar damage and neoangiogenesis. ${ }^{8,9}$ Other organs may also be affected by this form of thrombotic microangiopathy, resulting in multiple organ dysfunction syndrome and eventually death. ${ }^{10}$ Laboratory profiles seen in patients with severe COVID-19 (elevated D-dimers and fibrin degradation products [FDPs], and prolonged prothrombin time) are also consistent with a hypercoagulable state.

These authors are co-first authors.

published online January 22, 2021
Issue Theme Maintaining Hemostasis and Preventing Thrombosis in COVID-19 -Part II; Guest Editors: Emmanuel J. Favaloro, PhD, FFSc (RCPA) and Giuseppe Lippi, MD.

Address for correspondence Brandon Michael Henry, MD, The Heart Institute, Cincinnati Children's Hospital Medical Center, 3333 Burnet Ave., Cincinnati, OH 45229 (e-mail: brandon.henry@cchmc.org).

The role of the fibrinolytic system in CAC is not yet well established. It has been hypothesized that patients with severe COVID-19 have elevated levels of antifibrinolytic proteins, which create a hypofibrinolytic state, with subsequent failure of clot lysis. This hypothesis is supported by a recent thromboelastographic study by Wright and colleagues where a complete lack of lysis of clot at 30 minutes was reported in approximately $57 \%$ of ICU-admitted COVID-19 patients. ${ }^{11}$ Henry and colleagues recently demonstrated that ICU-admitted COVID-19 patients had lower levels of circulating plasminogen than non-ICU patients, suggesting an exhaustion of fibrinolysis. ${ }^{12}$ Recent small clinical studies have also demonstrated potential therapeutic effects of supplementing the fibrinolytic system, where exogenous administration of tissue plasminogen activator (tPA) resulted in improvement of respiratory function and reduced mortality in critically ill COVID-19 patients with acute respiratory distress syndrome (ARDS). ${ }^{13,14}$

Herein, we performed a prospective observational study to determine whether circulating plasma levels of plasminogen activator inhibitor-1 (PAI-1) and tPA may be significantly

(c) 2021. Thieme. All rights reserved. Thieme Medical Publishers, Inc., 333 Seventh Avenue, 18th Floor, New York, NY 10001, USA
DOI https://doi.org/ 10.1055/s-0040-1722308. ISSN 0094-6176. 
elevated in patients with severe COVID-19. We additionally determined the diagnostic performance of baseline PAI- 1 and tPA for predicting progression to severe disease.

Adults ( $>18$ years old) presenting to the University of Cincinnati Medical Center Emergency Department (ED) with symptoms of COVID-19 with a positive reverse transcriptionpolymerase chain reaction test for COVID-19 on nasopharyngeal swab were included. This study was approved by the Institutional Review Board of the University of Cincinnati. Blood samples were collected via routine draws for clinical indications in the ED. Circulating levels of TPA and PAI- 1 were determined using enzyme-linked immunosorbent assay (Technozym, Diapharma, West Chester, OH). The clinical course of these patients was then monitored for 30 days. The severity of COVID-19 at the time of presentation to the ED was the primary outcome of this study, while peak severity of COVID-19 within 30 days of index visit to the ED or hospital discharge was the secondary outcome. Severe disease was defined as a composite of (1) partial pressure of oxygen in arterial blood/fraction of inspired oxygen $\leq 300 \mathrm{~mm} \mathrm{Hg}$ or (2) patients requiring noninvasive ventilation using high flow nasal devices/mechanical ventilation/vital life support/ICU admission, or admission to the ICU.

Analysis of the data was performed using Prism 7 (GraphPad Software, San Diego, CA) and SPSS (IBM Statistics Software Version 25, Armonk, NY). Categorical data were reported as frequencies (\%) while continuous data were reported as median and interquartile range (IQR). Comparison of baseline tPA and PAI-1 levels between patients with and without severe COVID-19 at the time of index ED visit was performed using the Mann-Whitney U-test. The diagnostic performance of baseline tPA and PAI-1 for predicting peak 30-day severity of COVID-19 was assessed using receiver operating characteristic (ROC) curve, with calculation of the area under the curve (AUC) and its $95 \%$ confidence interval $(95 \% \mathrm{CI})$. Logistic regression was performed to estimate the effect of elevated tPA and PAI-1 ( $\geq 4$ and $>52.3 \mathrm{ng} / \mathrm{mL}$ respectively, based on ROC curve analysis) on the secondary outcome of peak 30-day severity of COVID-19, adjusting for age, sex, and comorbidities, and to calculate adjusted odds ratios (ORs) with the corresponding 95\% Wald Cl. Additionally, correlation between circulating levels of tPA/PAI- 1 and inflammatory biomarkers (interleukin [IL]-6, IL-10, tumor necrosis factor $\alpha$ [TNF $\alpha$ ], ferritin and Creactive protein $[\mathrm{CRP}]$ ) as well as platelet counts was performed using Spearman's correlation.

A total of 52 patients were included in this study. Of these, 46 had nonsevere disease at the time of index ED presentation, while six had severe disease. The median age in the severe group was 57 (IQR: 47-67) versus 50.5 (IQR: 37.8-66) years in the nonsevere group $(p=0.445)$. Males made up $83 \%$ of the severe group and $54.3 \%$ of the nonsevere group $(p=0.181)$. The comorbidities of these patients are presented in -Fig. 1A. Patients with severe disease had significantly higher levels of PAI-1 than those with the nonsevere disease (115.6 [IQR: 49.5-357] vs. 47.6 [IQR: 27.9-88.3] $\mathrm{ng} / \mathrm{mL} ; p=0.045$ ) (-Fig. 1B). Circulating tPA levels were also elevated in patients with severe disease compared with those with nonsevere disease, although this did not achieve statistical significance
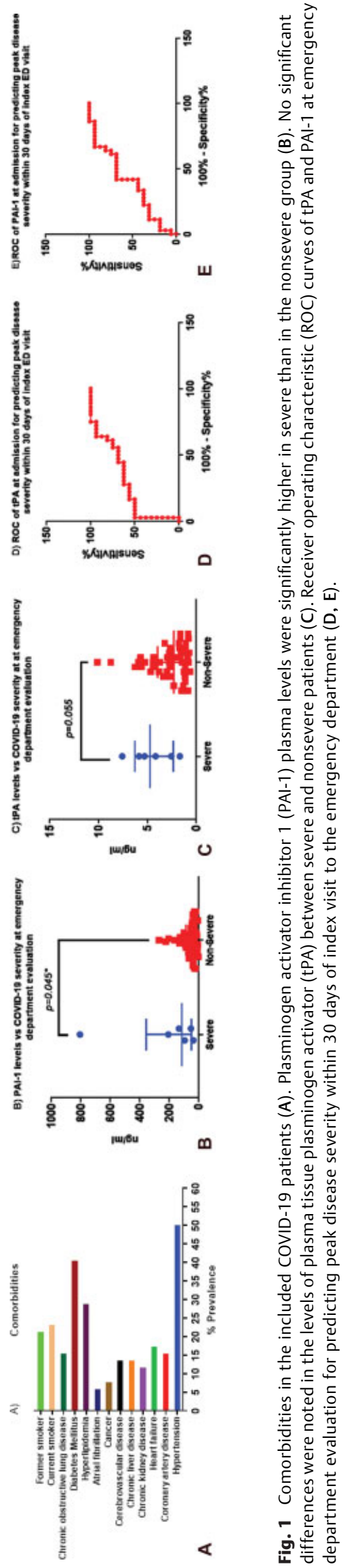
Table 1 Age-, sex-, and comorbidity-adjusted multivariable logistic regression for $\mathrm{tPA}$ and PAl-1 as independent predictors of severe COVID-19 within 30 days

\begin{tabular}{|l|l|l|l|l|}
\hline Variable & Estimate & $\begin{array}{l}\text { Standard } \\
\text { error }\end{array}$ & $\begin{array}{l}\text { Odds ratio } \\
(95 \% \mathrm{Cl})\end{array}$ & $p$-Value \\
\hline $\begin{array}{l}\text { Elevated } \\
\text { PAA }\end{array}$ & 1.897 & 0.788 & $\begin{array}{l}6.67 \\
(1.42-31.21)\end{array}$ & $0.016^{\mathrm{a}}$ \\
\hline $\begin{array}{l}\text { Elevated } \\
\text { PAl-1 }\end{array}$ & 2.053 & 0.897 & $\begin{array}{l}7.79 \\
(1.34-45.21)\end{array}$ & $0.022^{\mathrm{a}}$ \\
\hline
\end{tabular}

Abbreviations: $\mathrm{Cl}$, confidence interval; PAl-1, plasminogen activator inhibitor 1; tPA, tissue plasminogen.

${ }^{\mathrm{a}}$ Significant at the value of 0.05 .

(4.7 [IQR: 2.4-6.3] vs. 2.3 [IQR: $1.2-4.0] \mathrm{ng} / \mathrm{mL} ; p=0.055$ ) (-Fig. 1C). A total of 16 patients developed severe disease within 30 days of index ED presentation. ROC curves were generated for TPA and PAI- 1 at the time of initial ED evaluation for predicting peak disease severity within 30 days of index ED visit. The AUC of tPA was 0.73 (95\% CI: $0.58-0.89 ; p=0.008$ ). Analysis of ROC data determined that a tPA cut-off of $\geq 4 \mathrm{ng} / \mathrm{mL}$ was associated with 0.56 sensitivity and 0.83 specificity, respectively ( - Fig. 1D). The AUC for PAI-1 was 0.63 (95\% CI: $0.47-0.79 ; p=0.142)$, with $\geq 52.3 \mathrm{ng} / \mathrm{mL}$ cut-off displaying 0.69 sensitivity and 0.58 specificity ( - Fig. 1E). Multivariable logistic regression (adjusted for age, sex, and comorbidities) for tPA and PAI- 1 levels above the ROC cut-offs (i.e., $\geq 4$ and $\geq 52.3 \mathrm{ng} / \mathrm{mL}$, respectively) as independent predictors of peak 30-day severity revealed that elevated tPA and PAI-1 levels were associated with increased odds of developing severe disease ( $\mathrm{OR}=6.67,95 \% \mathrm{CI}: 1.42-31.21, p=0.016$ and $\mathrm{OR}=7.79,95 \% \mathrm{CI}: 1.34-45.21, p=0.022$, respectively) (-Table 1). Circulating tPA positively correlated with IL-10 $(r=0.431,95 \%$ CI: 0.168-0.637; $p=0.002)$, TNF $\alpha(r=0.424$, 95\% CI: $0.159-0.631 ; p=0.002)$, serum ferritin $(r=0.471,95 \%$ CI: $0.219-0.664 ; p<0.001)$, and lactate dehydrogenase $(r=0.443,95 \%$ CI: $0.183-0.641 ; p<0.001)$, while PAI- 1 positively correlated with IL-10 $(r=0.318,95 \% \mathrm{CI}: 0.038-0.551$; $p=0.023)$, TNF $\alpha(r=0.310,95 \%$ CI: $0.029-0.546 ; p=0.027)$, and platelet counts ( $r=0.347,95 \% \mathrm{Cl}: 0.061-0.058 ; p=0.016)$ (-Table 2).

Taken together, these findings suggest that the fibrinolytic system plays a significant role in the pathophysiology of COVID-19. In normal lung physiology, the pulmonary alveolar space (PAS) is thought to have a profibrinolytic milieu. ${ }^{15}$ Previous studies demonstrated that suppression of the fibrinolytic system is a key feature of non-COVID-19 ARDS. ${ }^{16,17}$ PAI-1 may be produced by alveolar macrophages, with elevated levels during lung injury and inflammation. ${ }^{18}$ Moreover, the observed positive correlation between PAI-1 levels and platelet counts suggests that platelets are a substantial source of PAI-1 in these patients. Finally, genetic ${ }^{19}$ and metabolic factors (increased abdominal adipose tissue) ${ }^{20}$ may also influence increased production of PAI-1.

The findings of elevated PAI- 1 levels in patients with severe COVID-19 as seen in the current and one other study, ${ }^{21}$ combined with recent thromboelastographic investigations,

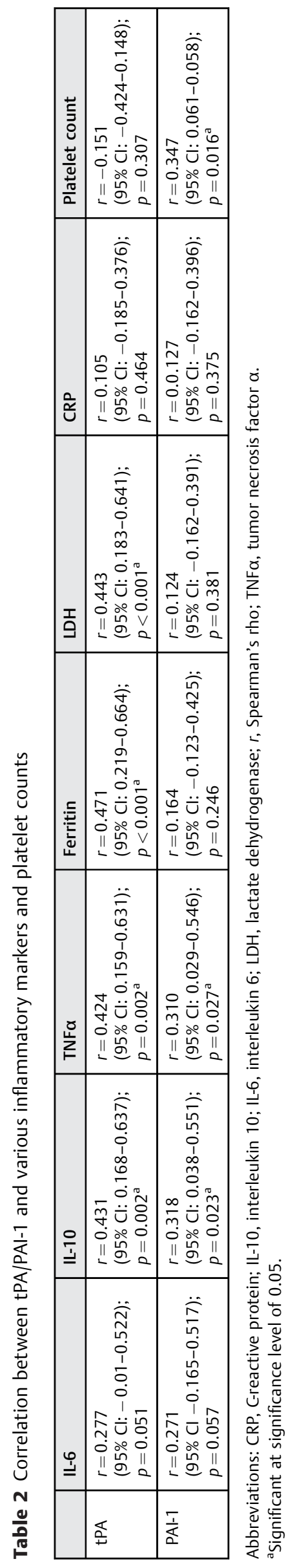


suggest dysregulation of the fibrinolytic system and a phenomenon of "fibrinolytic shutdown" or "temporary exhaustion" in COVID-19 patients. ${ }^{11,21}$ These phenomena would result in reduced clot lysis and persistence of thrombi within the capillary network. The presence of "fibrinolytic shutdown" seems to be inconsistent with the observations of elevated D-dimer levels in patients with severe COVID-19, ${ }^{22}$ since formation of D-dimers and FDP generation would need an intact and efficient fibrinolytic system. This discrepancy can, however, be attributed to temporal changes in levels of tPA and PAI-1, as well as to insufficient hyperfibrinolysis characterized by systemic suppression and local (i.e., within the pulmonary tissue) hyperactive fibrinolysis derangement, as recently pinpointed by Ji et al. ${ }^{23}$ This is in concordance with recent observations by Henry et al where COVID-19 patients progressing to severe disease had lower levels of plasminogen compared with discharged patients. ${ }^{12}$ This suggests that the high D-dimer levels observed in patients with severe COVID19 may come from an initial rise in tPA/plasmin. This hypothesis is further supported by observations by Wright and colleagues where patients who met the criteria of hypofibrinolysis based on thromboelastographic findings had a marked decline in D-dimer levels after an initial rise. ${ }^{11}$ Our findings also suggest that elevated tPA and PAI- 1 are associated with poor outcomes among COVID-19 patients, consistent with previous reports.

In their preclinical study on nonhuman primates, Biemond and colleagues ${ }^{24}$ demonstrated that a bolus injection of bacterial endotoxin resulted in rapid increase in tPA and plasmin levels within the first 2 hours. PAI-1 levels remained constant during this period, after which a rapid increase was observed with simultaneous decrease in TPA and plasmin activities. The authors noted that the fibrinolytic system was suppressed for the remainder of the study due to high PAI- 1 levels. ${ }^{24}$ Thus, we suspect in COVID-19 that the initial rise in TPA is driven by inflammation, after which remnant tPA is complexed to PAI-1 and rendered inactive, while any subsequent tPA released is insufficient to overcome the increase in circulating PAI-1.

Since upregulation of PAI-1 levels is a common feature of thrombotic microangiopathies (TMAs) such as primary and secondary thrombotic thrombocytopenic purpura and hemolytic uremic syndrome, ${ }^{25-27}$ the observations of the current study support the presence of a TMA phenomenon in COVID-19. This is further supported by the positive correlation observed between PAI-1 and proinflammatory cytokines such as TNF $\alpha$, which are important mediators of endothelial damage, a hallmark of TMA. When combined with previous observations of reduced ADAMTS13 (a disintegrin and metalloproteinase with a thrombospondin type 1 motif, member 13) in COVID-19, a picture strongly suggestive of TMA-like phenomenon emerges. ${ }^{28}$

Our study was limited by the small sample size. In the analysis of PAI-1, we noted an outlier among the six severe cases that could have biased the statistical finding. This was however delimited by ROC and regression analysis for 30-day peak COVID-19 severity in which there were more patients in the severe group (16 patients) with no obvious outliers. We measured circulating plasma tPA and PAI-1, which may not be reflective of the actual levels within the PAS. Further, we did not perform serial measurements of PAI-1/tPA. Future studies should focus not only on circulating but also on local (pulmonary) levels of tPA/PAI-1, as well as their temporal changes along the clinical course of COVID-19 patients to provide further clarification of changes in the fibrinolytic system in COVID-19 patients.

In conclusion, patients with severe COVID-19 have significantly elevated plasma levels of PAI-1. Elevated levels of tPA and PAI- 1 are independent predictors of progression to severe COVID-19. The evidence provided suggests that fibrinolytics should continue to be investigated as therapeutic avenue in patients with COVID-19.

\section{Funding}

This study was funded by the University of Cincinnati College of Medicine Special Coronavirus (COVID-19) Research Pilot Grant Program.

Conflict of Interest

None declared.

\section{References}

1 Becker RC. COVID-19 update: Covid-19-associated coagulopathy.J Thromb Thrombolysis 2020;50(01):54-67

2 Panigada M, Bottino N, Tagliabue P, et al. Hypercoagulability of COVID-19 patients in intensive care unit: a report of thromboelastography findings and other parameters of hemostasis. J Thromb Haemost18(07):1738-1742

3 Violi F, Pastori D, Cangemi R, Pignatelli P, Loffredo L. Hypercoagulation and antithrombotic treatment in coronavirus 2019: a new challenge. Thromb Haemost 2020;120(06):949-956

4 Henry BM, Vikse J, Benoit S, Favaloro EJ, Lippi G. Hyperinflammation and derangement of renin-angiotensin-aldosterone system in COVID-19: a novel hypothesis for clinically suspected hypercoagulopathy and microvascular immunothrombosis. Clin Chim Acta 2020;507:167-173

5 Bray MA, Sartain SE, Gollamudi J, Rumbaut RE. Microvascular thrombosis: experimental and clinical implications. Transl Res 2020;225:105-130

6 Klok FA, Kruip MJHA, van der Meer NJM, et al. Incidence of thrombotic complications in critically ill ICU patients with COVID-19. Thromb Res 2020;191:145-147

7 Cheruiyot I, Kipkorir V, Ngure B, Misiani M, Munguti J, Ogeng'o J. Arterial thrombosis in coronavirus disease 2019 patients: a rapid systematic review. Ann Vasc Surg 2021;70:273-281

8 Ackermann M, Verleden SE, Kuehnel M, et al. Pulmonary vascular endothelialitis, thrombosis, and angiogenesis in Covid-19. N Engl J Med 2020;383(02):120-128

9 Carsana L, Sonzogni A, Nasr A, et al. Pulmonary post-mortem findings in a series of COVID-19 cases from northern Italy: a twocentre descriptive study. Lancet Infect Dis 2020;20(10):1135-1140

10 Ciceri F, Beretta L, Scandroglio AM, et al. Microvascular COVID-19 lung vessels obstructive thromboinflammatory syndrome (MicroCLOTS): an atypical acute respiratory distress syndrome working hypothesis. Crit Care Resusc 2020;22(02):95-97

11 Wright FL, Vogler TO, Moore EE, et al. Fibrinolysis shutdown correlation with thromboembolic events in severe COVID-19 infection. J Am Coll Surg 2020;231(02):193.e1-203.e1

12 Henry BM, Benoit SW, Hoehn J, Lippi G, Favaloro EJ, Benoit JL. Circulating plasminogen concentration at admission in patients with coronavirus disease 2019 (COVID-19). Semin Thromb Hemost 2020;46(07):859-862 
13 Wang J, Hajizadeh N, Moore EE, et al. Tissue plasminogen activator (tPA) treatment for COVID-19 associated acute respiratory distress syndrome (ARDS): a case series. J Thromb Haemost 2020;18 (07):1752-1755

14 Choudhury R, Barrett CD, Moore HB, et al. Salvage use of tissue plasminogen activator (tPA) in the setting of acute respiratory distress syndrome (ARDS) due to COVID-19 in the USA: a Markov decision analysis. World J Emerg Surg 2020;15(01):29

15 Medcalf RL, Keragala CB, Myles PS. Fibrinolysis and COVID-19: a plasmin paradox. J Thromb Haemost 2020;18(09):2118-2122

16 Idell S, James KK, Levin EG, et al. Local abnormalities in coagulation and fibrinolytic pathways predispose to alveolar fibrin deposition in the adult respiratory distress syndrome. J Clin Invest 1989;84(02):695-705

17 Ozolina A, Sarkele M, Sabelnikovs O, et al. Activation of coagulation and fibrinolysis in acute respiratory distress syndrome: a prospective pilot study. Front Med (Lausanne) 2016; 3:64

18 Chapman HA, Yang XL, Sailor LZ, Sugarbaker DJ. Developmental expression of plasminogen activator inhibitor type 1 by human alveolar macrophages. Possible role in lung injury. J Immunol 1990;145(10):3398-3405

19 Väisänen SB, Humphries SE, Luong LA, Penttilä I, Bouchard C, Rauramaa R. Regular exercise, plasminogen activator inhibitor-1 (PAI-1) activity and the 4G/5G promoter polymorphism in the PAI-1 gene. Thromb Haemost 1999;82(03):1117-1120

20 Alessi MC, Peiretti F, Morange P, Henry M, Nalbone G, Juhan-Vague I. Production of plasminogen activator inhibitor 1 by human adipose tissue: possible link between visceral fat accumulation and vascular disease. Diabetes 1997;46(05):860-867

21 Nougier C, Benoit R, Simon M, et al. Hypofibrinolytic state and high thrombin generation may play a major role in sars-cov2 associated thrombosis. J Thromb Haemost 2020;18(09):2215-2219

22 Lippi G, Favaloro EJ. D-dimer is associated with severity of coronavirus disease 2019: a pooled analysis. Thromb Haemost 2020;120(05):876-878

$23 \mathrm{JiH}-\mathrm{LJ}$, Su Z, Zhao R, et al. Insufficient hyperfibrinolysis in COVID-19: a systematic review of thrombolysis based on meta-analysis and meta-regression. medRxiv. Doi: 10.1101/2020.09.07.20190165

24 Biemond BJ, Levi M, Ten Cate $\mathrm{H}$, et al. Plasminogen activator and plasminogen activator inhibitor I release during experimental endotoxaemia in chimpanzees: effect of interventions in the cytokine and coagulation cascades. Clin Sci (Lond) 1995;88(05): 587-594

25 Tersteeg C, Joly BS, Gils A, et al. Amplified endogenous plasmin activity resolves acute thrombotic thrombocytopenic purpura in mice. J Thromb Haemost 2017;15(12):2432-2442

26 Anthony MT, Zeigler ZR, Lister J, et al. Plasminogen activator inhibitor (PAI-1) antigen levels in primary TTP and secondary TTP postbone marrow transplantation. Am J Hematol 1998;59(01):9-14

27 Martin AA, Woolven BL, Harris SJ, et al. Plasminogen activator inhibitor type-1 and interleukin-6 in haemolytic uraemic syndrome. J Paediatr Child Health 2000;36(04):327-331

28 Martinelli N, Montagnana M, Pizzolo F, et al. A relative ADAMTS13 deficiency supports the presence of a secondary microangiopathy in COVID 19. Thromb Res 2020;193:170-172 